Artículo Original

REV EXP MED

\section{Clima laboral y satisfacción del profesional de enfermería en el servicio de emergencias de un hospital público de Chiclayo, Perú}

Susan Miriam Oblitas-Guerrero ${ }^{1,2, a}$

\title{
RESUMEN
}

Objetivo. Determinar la relación del clima laboral y la satisfacción del profesional de enfermería en el servicio de emergencias del Hospital Regional Lambayeque (HRL). Chiclayo, Perú, 2018. Material y métodos. Estudio transversal en 42 profesionales del servicio de emergencia del HRL. Para la recolección de datos se aplicó un cuestionario de clima laboral y otro de satisfacción laboral según Herzberg, adaptado por la investigadora. Resultados. La población se caracterizó por ser de sexo femenino (92,9\%), edad entre los 20 a 35 años, casadas ( $42,9 \%)$, grado de maestro $(21,4 \%$ ) y con especialidad en emergencia y desastres (92,9\%). Respecto al clima laboral, un $66,7 \%$ lo percibe promedio, el $21,4 \%$ saludable y el $11,9 \%$ restante lo percibe no favorable. Respecto a la satisfacción laboral, un $66,7 \%$ presenta un grado moderado de satisfacción, el $28,6 \%$ un grado alto y el 4,8 \% restante un grado bajo. Se observó relación entre el clima y satisfacción laboral $(p=0,002)$. Conclusiones. Existe relación significativa del clima laboral en la satisfacción del profesional de enfermería del HRL en el periodo de estudio, por lo que es importante fortalecer las condiciones de trabajo de estos profesionales de la salud.

Palabras Clave: Condiciones de Trabajo, Satisfacción laboral, Enfermeras y Enfermeros, Enfermeras Especialistas (Fuente: DeCS-BIREME).

\section{Laboral climate and satisfaction of emergency nurses in a public hospital in Chiclayo, Peru}

\section{ABSTRACT}

Objective. To determine the relationship between the work climate and the satisfaction of the emergency nurses of the Hospital Regional Lambayeque (HRL). Chiclayo, Peru, 2018. Material and methods. Cross-sectional study of 42 nurses from the HRL emergency service. For data collection, a work climate questionnaire and a job satisfaction questionnaire were applied according to Herzberg, adapted by the researcher. Results. The population was characterized by being female (92.9\%), age between 20 to 35 years, married (42.9\%), Master degree (21.4\%) and specializing in emergencies and disasters (92, $9 \%)$. Regarding the work climate, $66.7 \%$ perceive it as average, $21.4 \%$ healthy and the remaining $11.9 \%$ perceive it as unfavorable. Regarding job satisfaction, $66.7 \%$ have a moderate degree of satisfaction, $28.6 \%$ a high grade and the remaining $4.8 \%$ a low grade. A relationship between climate and job satisfaction was observed ( $p=0.002$ ). Conclusions. There is a significant relationship between the work environment in the satisfaction of HRL nurses in the study period, so it is important to strengthen the working conditions of these health professionals.

Keywords: Working climate, Job satisfaction, Nurses, Nursing Assistants (Source: MeSH-NLM).

\footnotetext{
${ }^{1}$ Servicio de emergencias del Hospital Regional Lambayeque. Chiclayo, Perú.

${ }^{2}$ Grupo de Investigación Enfermería Social y Cuidado a la Persona Adulta-GIESCA, Universidad Católica Santo Toribio de Mogrovejo. Chiclayo, Perú.

a Enfermera, especialista en Emergencias. Maestra en ciencias con mención en Gerencia en Servicios de Salud.
} 


\section{INTRODUCCIÓN}

En la actualidad existe una preocupación creciente en los sistemas de salud del mundo sobre la situación laboral de las enfermeras y enfermeros, la Organización Mundial de Salud (OMS), identifica este recurso humano como eje en la respuesta de los sistemas de salud a las condiciones epidemiológicas futuras de las poblaciones ${ }^{(1)}$.

A pesar que diversas organizaciones internacionales como la OMS y la organización internacional de trabajo (OIT), manifiestan que las personas son el más preciado capital del que disponen las organizaciones, más aún aquellas organizaciones, dedicadas al cuidado de la salud, en las que los profesionales desarrollan su actividad en un íntimo contacto, con personas que se encuentran en una situación de especial vulnerabilidad debido a su enfermedad (2).

Las instituciones de salud no otorgan la suficiente relevancia en los temas de clima laboral y satisfacción laboral, donde el recuro humano muestran malestar en el ambiente laboral, aspecto que se relaciona en la satisfacción personal y profesional (3). Consiguientemente, los responsables de dirigir y administrar las organizaciones de salud no están enteramente convencidos que una manera eficaz de mantener satisfechos a los trabajadores para lograr un mejor rendimiento y obtener resultados deseables es garantizando un clima laboral adecuado ${ }^{(4)}$.

Investigaciones internacionales tanto en Europa como en Asia señalan que la satisfacción laboral se usa para medir qué tan contento está un empleado con el trabajo. La alta satisfacción laboral puede mejorar el entusiasmo del personal y es beneficioso para el éxito y el progreso de la organización. Puede llevar a una menor rotación y un servicio de alta calidad. El personal de salud con baja satisfacción laboral puede sufrir problemas médicos y la salud individual de los empleados puede influir en la estabilidad general del personal de salud. Los empleados insatisfechos son más propensos a abandonar la organización y, como resultado, los empleados restantes pueden dedicarse a actividades contraproducentes tales como un servicio de baja calidad y causar daños al equipo ${ }^{(5)}$.

La realidad mundial sobre la situación laboral de las enfermeras y enfermeros, no es ajeno a la coyuntura del Perú. Donde el clima laboral es autoritario, inflexible con el recurso humano generando insatisfacción y desmotivación laboral. La OIT reconoce que el entorno laboral puede ser un agente capaz de deteriorar la salud de las personas durante el desempeño de su trabajo ${ }^{(6)}$ y entre las características de dicho entorno, los factores psicosociales y organizativos desempeñan un valor relevante ${ }^{(7,8)}$.

Las investigaciones en el ámbito de la enfermería resalcan relaciones significativas de la satisfacción laboral con variables como: un bajo clima laboral, sobrecarga de trabajo, entornos de trabajo inadecuados, entre otras ${ }^{\left({ }^{8}\right)}$. Donde el recurso humano se encuentra desfavorecido por múltiples factores siendo uno de ellos las reformas sectoriales, cuya desatención en los últimos años ha provocado consecuencias negativas en el desarrollo de la atención de salud ${ }^{(9)}$. Donde las condiciones de trabajo, tanto de los profesionales de salud como de los equipos de apoyo (técnico y auxiliar), son esenciales para la entrega segura de servicios de calidad (10).

Siendo un desafío actual en el área de la gestión de personas que trabajan en organizaciones sanitarias, quien buscan establecer mecanismos de planificación de recursos humanos, para que puedan cumplir efectivamente con las demandas de atención de la salud y, al mismo tiempo, proporcionar estabilidad laboral a los trabajadores con el fin de satisfacer dicha atención ${ }^{(11)}$.

La presente investigación centra su estudio en dos constructos: clima laboral y satisfacción laboral en el profesional de enfermería, los cuales fueron respaldados en la teoría de clima laboral de Litwin y Stringer y la teoría de los dos factores o teoría bifactorial de la satisfacción Herzberg. El clima laboral y la satisfacción laboral son dos constructos distintos, pero relacionados; el primero hace referencia a la percepción que el individuo tiene respecto a la estructura y las interacciones psicosociales en su ambiente de trabajo ${ }^{(8)}$, mientras que el segundo se enfoca en las actitudes y percepciones que tienen los individuos hacia su trabajo ${ }^{(12)}$.

Uceda en el año 2017 en trabajadores de un dentro de salud de Chiclayo, donde encontró que $72,1 \%$ de los usuarios internos presenta un clima laboral medianamente favorable, el 25,6 \% favorable. En cuanto a la satisfacción laboral el $86 \%$ de los usuarios internos consideran que la satisfacción está en un nivel medio; concluyendo que sí existe relación directa entre clima laboral y satisfacción laboral ${ }^{(13)}$ Asimismo, Chiquinta, en el año 2017, también en Chiclayo, realizó una investigación en enfermeras del servicio de emergencia del Hospital Regional Lambayeque Chiclayo; donde reportó que el valor del clima organizacional fue de 74,33 el cual corresponde a la calificación "por mejorar"; la satisfacción laboral está determinado por un valor promedio 133.00 el cual corresponde a la calificación de "regular y el valor de la correlación de las variables es 0.73 , el cual indica que existe una correlación positiva alta ${ }^{(14)}$.

Por esta razón, se determinó la relación del clima laboral y la satisfacción de las enfermeras de emergencia del Hospital Regional Lambayeque (HRL). Chiclayo, Perú, durante el 2018. 


\section{MATERIAL Y MÉTODOS}

Diseño de estudio.

El diseño de estudio de esta investigación es de tipo descriptivo-correlacional.

\section{Escenario.}

El presente estudio se desarrolló en el Hospital Regional Lambayeque, MINSA-Chiclayo de Nivel III-1, máximo nivel de los establecimientos de salud en el país, debido a sus avances tecnológicos y alta especialización de los servicios, por lo que es un centro referencial para pacientes de toda la macro región Norte. Cuenta con un servicio de emergencia que atiende las 24 horas, compuesta por triaje, unidad de trauma shock, cuatro tópicos: cirugía y traumatología, medicina, pediatría y ginecología, y con la unidad de observación pediátrica y de adultos.

\section{Población y muestra}

La población estuvo conformado por 42 profesionales de enfermería pertenecientes al servicio de emergencia del Hospital Regional de Lambayeque, de condición homogénea, ya que la población tuvo las mismas características según los criterios de inclusión, considerando la especificación y tamaño de la población. El muestreo fue no probabilístico.

\section{Variables e instrumentos de medición}

Cuestionario de clima laboral: Es un instrumento basado en el cuestionario del Ministerio de Salud, en el año 2009 (10,15), y el cuestionario de clima laboral Litwin y Stringer modificado y adaptado por la investigadora. Dicho instrumento tiene por objetivo identificar el clima laboral de los profesionales de salud en el contexto hospitalario. Este instrumento consta de 27 preguntas que miden tres dimensiones del clima laboral: diseño organizacional, potencial humano y cultura de la organización. Se calificó de la siguiente manera: clima favorable, clima promedio y clima no favorable.

Cuestionario satisfacción laboral según Herzberg: Es un instrumento basado en el cuestionario satisfacción laboral según Herzberg desarrollado por Gutiérrez en el año 2015 (16), el cual ha sido modificado y adaptado por la investigadora basada en los planteamientos teóricos de Frederick Herzberg. Dicho instrumento tiene por objetivo identificar la satisfacción laboral de los profesionales de la salud en el contexto hospitalario. Este instrumento consta de 24 preguntas y evaluó las dimensiones: Motivacional o intrínseca e Higiénica o extrínseca. Clasificación: alta satisfacción laboral, moderada satisfacción laboral y baja satisfacción laboral.

\section{Validez de los instrumentos}

Validez de contenido: Se realizó mediante la calificación por medio de criterio de expertos, cinco en total, acerca de la pertinencia de los reactivos en relación al constructo evaluado por dimensiones. Para efectos de este análisis, se utilizó para la evaluación de la validez de contenido la fórmula de $\mathrm{V}$ de Aiken. El coeficiente $\mathrm{V}$ de Aiken total es mayor de 0,69 , este valor indica que instrumento es válido para recabar información.

Confiabilidad: Se determinó a través de la aplicación de la prueba piloto a una población con características similares a la población de estudio, en un total de 10 enfermeras. Se utilizó para la evaluación de la confiabilidad el coeficiente Alfa de Cronbach, en él tuvo mayor a 0,70.

Análisis estadístico de los datos

El procesamiento y análisis estadístico se realizó mediante la clasificación, ordenamiento y codificación de datos mediante el uso del programa estadístico: SPSS/info/ software versión 23.0. Descriptivo: Los resultados están representados en tablas simples y de doble entrada, gráficos, cifras absolutas y relativas. Asimismo, se aplicó la prueba estadística de independencia de criterios (Chi2) para determinar relación de las variables de estudio, con un nivel de significancia de $p<0.05$.

\section{Consideraciones éticas}

El trabajo de investigación se presentó en primer lugar a la Escuela de Post grado de la Universidad Nacional Pedro Ruiz Gallo, una vez aprobada se solicitó la autorización a la dirección de investigación del Hospital Regional, dando curso regular para la aplicación del instrumento a las enfermeras del servicio de emergencia. La participación en el estudio fue voluntario a través de la firma de un consentimiento informado.

\section{RESULTADOS}

El servicio de emergencia del Hospital Regional de Lambayeque, la mayoría de los profesionales de enfermería son de sexo femenino (92,9\%), de edades entre los 20 a 35 años, el $42,9 \%$ so casadas, el $35,7 \%$ solteras, el $21,4 \%$ tienen grado de maestro, el 92,9\% cuentan con especialidad en emergencia y desastres. El $61,9 \%$ presenta un tiempo de servicio de 1 a 4 años y el $23,8 \%$ tiene más de 5 años. Cerca del $50 \%$ de los profesionales se encuentran nombrados ( $47,6 \%)$; sin embargo, el $7,1 \%$ se encuentra en condición de servicios no personales (SNP) el $40,5 \%$ son docentes universitarios dentro de la Región Lambayeque. Conocedores el perfil laboral y personal de las enfermeras del servicio de emergencia HRL a continuación, se presenta los resultados de las variables en estudio.

En la tabla 1, se presenta el clima laboral de las enfermeras que laboran en el servicio de emergencia del Hospital Regional Lambayeque. Donde se observa que, de una distribución de 42 profesionales de enfermería, el 66,7\% percibe un clima laboral promedio, el $21,4 \%$ percibe un clima laboral favorable, el $11,9 \%$ restante de enfermeros 
considera que un clima laboral en el servicio no favorable.

En la tabla 2, se presenta el grado de satisfacción laboral de las enfermeras que laboran en el servicio de emergencia del HRL. Donde se observa que, de una distribución de 42 profesionales de enfermería el $66,7 \%$ presenta un grado moderado de satisfacción, el $28,6 \%$ un grado alto de satisfacción y el $4,8 \%$ restante un grado bajo de satisfacción laboral. El análisis bivariado entre el clima laboral y la satifacción de las enfermeras mostró relación entre ellas (tabla 3).

Tabla 1. Clima laboral de las enfermeras que laboran en el servicio de emergencia del Hospital Regional Lambayeque, Chiclayo, 2018.

\begin{tabular}{lcc} 
Clima laboral & $\mathrm{N}$ & $\%$ \\
Favorable & 9 & 21,4 \\
Promedio & 28 & 66,7 \\
No favorable & 5 & 11,9 \\
Total & 42 & 100 \\
\hline \hline
\end{tabular}

Tabla 2. Satisfacción laboral de las enfermeras que laboran en el servicio de emergencia del Hospital Regional Lambayeque, Chiclayo, 2018.

\begin{tabular}{lcc} 
Grado de satisfacción laboral & $\mathrm{N}$ & $\%$ \\
Alta & 12 & 28,6 \\
Moderada & 28 & 66,7 \\
Baja & 2 & 4,8 \\
Total & 42 & 100 \\
\hline \hline
\end{tabular}

Tabla 3. Relación del clima laboral y la satisfacción de las enfermeras del servicio de emergencia del Hospital Regional Lambayeque, Chiclayo, 2018.

\begin{tabular}{lcccc} 
Clima laboral & \multicolumn{3}{c}{ Satisfacción laboral } & Valor de p \\
& Alta & Moderada & Baja & \\
Favorable & 7 & 2 & 0 & \\
Promedio & 5 & 22 & 1 & 0,002 \\
No favorable & 0 & 4 & 1 & \\
\hline \hline
\end{tabular}

\section{DISCUSION}

Los resultados obtenidos en diversas investigaciones guardan relación con los hallazgos de Chávez y Ríos3 y Chiquinta ${ }^{(14)}$ quienes manifiestan que más del $50 \%$ de los profesionales de enfermería en el ámbito hospitalario perciben un clima laboral promedio con un 69,0 \% y 74,3 \% respectivamente en cada estudio.

Lo que permite inferir que el clima laboral en el servicio de emergencia no es el más óptimo dado la coyuntura del propio servicio, sin embargo, el equipo de salud y el profesional de enfermería maneja la situación e intenta adaptarse con el objeto de brindar un servicio de salud que beneficie al usuario externo. Lo cual se ve reflejado en el análisis de los ítems el profesional de enfermería quienes están de acuerdo en que el personal de salud del servicio de emergencia saca adelante la atención de salud con creatividad, aun cuando las condiciones logísticas y laborales no son las adecuadas.

Además, están totalmente de acuerdo que les interesa el desarrollo de su institución y que están comprometidos con el mismo y que se sienten orgullosos de pertenecer a esta institución de salud. Además, considera que la coordinadora o jefe de servicio trata de obtener información del equipo o staff antes de tomar una decisión.

No obstante, a pesar que el profesional de enfermería se siente identificado y comprometido con la institución de salud específicamente con su servicio. Existe un porcentaje minoritario pero significativo de profesionales de enfermería que perciben un clima laboral no favorable $(11,9$ $\%)$. Resultados que guardan relación con los hallazgos Montalvo ${ }^{(9)}$ quien a través de su estudio "Clima organizacional en trabajadores de un hospital general de Ica" obtuvo que $12,4 \%$ de profesionales percibieron un clima no favorable.

La percepción que el trabajador tiene de las estructuras y procesos organizacionales y de las interacciones entre características personales y organizacionales que afectan, directa o indirectamente, el comportamiento de las personas dentro de una organización.

Los resultados obtenidos coinciden con los hallazgos Chiquinta ${ }^{(14)}$ quien a través de su estudio "Clima Organizacional y Satisfacción laboral de las enfermeras en el servicio de Emergencia del Hospital Regional Lambayeque." Concluyó que existe una correlación positiva considerable entre el clima organización y la satisfacción [Rho=0,73 $p<0,005$ ] cabe señalar que los resultados obtenido en este estudio fueron evaluados con otros instrumentos distintos al desarrollado de la presente investigación, pero con el mismo enfoque teórico. Lo que permite señalar que independientemente de los instrumentos el clima laboral guarda una estrecha relación con la satisfacción laboral.

Los resultados obtenidos en la presente investigación afirman que el clima laboral influye significativamente en la satisfacción laboral, lo cual es confirmado por Gago et al. ${ }^{(8)}$; quien consideran que el clima laboral es una variable que influye positivamente en la satisfacción laboral del personal de enfermería y tiene un impacto en los niveles de estrés, ansiedad, depresión y un rol relevante en el desarrollo de agotamiento ${ }^{(8)}$.

Montoya, en su investigación señala que resulta impor- 
tante que los empleados y trabajadores expresen su opiniones sobre el desenvolvimiento en la organización y cómo se sienten al desempeñarse en ella; constituyéndose así un instrumento de indagación que funciona bajo la premisa de generar beneficios cuando se implementan acciones correctivas en los aspectos que lo requieran; consecuentemente, constituyen un excelente mecanismo para conocer de manera indirecta la calidad de gestión en la organización (17).

El presente estudio tuvo limitaciones, por tratarse de una población de estudio limitada y restringida a un solo hospital, los resultados no son extrapolables a otras poblaciones de enfermeras.

El personal de enfermería del servicio de emergencia del Hospital Regional Lambayeque durante el 2018, presentó predominio de clima y satisfacción laboral medios, asimismo, existe relación significativa del clima laboral en la satisfacción en la población estudiada, por lo que es importante fortalecer las condiciones de trabajo de estos profesionales de la salud.

Declaración de conflictos de interés. Enfermera que trabaja en el servicio de emergencia.

Fuente de financiamiento. Autofinanciamiento.

Agradecimiento. Se agradece a todas mis compañeras del servicio de Emergencia, por las facilidades para la recolección de datos durante el estudio.

\section{REFERENCIAS BIBLIOGRÁFICAS}

1. Nigenda G, Ruiz J, Rosales Y, Bejarano R. Enfermeras con licenciatura en México: estimación de los niveles de deserción escolar y desperdicio laboral. salud publica mex. 2006; 48(1), 22-29.

2. Villar J, León J. Condiciones de trabajo y calidad de vida laboral en profesionales de la salud: el papel modulador de la resiliencia y la autoeficacia, sobre el síndrome de burnout y el engagement [Tesis doctoral]. Ica: Departamento de Psicología Social de la Universidad de Sevilla; 2015.

3. Chavez D, Rios K. Clima organizacional y satisfacción laboral de las enfermeras en el servicio de cirugía hospital Víctor Lazarte Echegaray [Tesis para obtener el título profesional de Licenciada en enfermería]. Trujillo: Universidad Privada Antenor Orrego; 2015

4. Chiavenato I. Políticas Administrativas y Administración de Recursos Humanos $5^{\circ}$ Edición. Editorial Bogotá: McGraw Hill. 2010.

5. Yong L, Xiao-Min H, Xiao-Liang H, Xiao-Dong Z, Pi G, Li-Fen F et al. Job satisfaction and associated factors among healthcare staff: a cross-sectional study in Guangdong Province, China. BMJ. 2016; 6(7): e011388. doi: 10.1136/bmjopen-2016-011388.

6. Houtman I, Kompier M. Trabajo y salud mental. En OIT, Enciclopedia de salud y seguridad en el trabajo. Madrid: OIT (ED). 2001.

7. Gil-Monte P. El Síndrome de quemarse por el Trabajo (Burnout). Una Perspectiva histórica. Madrid: Ediciones Pirámide. 2005

8. Gago K, Martínez I, Alegre A. Clima laboral y síndrome de burnout en enfermeras de un hospital general de Huancayo, Perú. PSIENCIA Revista Latinoamericana de Ciencia Psicológica. 2017; 9(4):1-15. doi:10.5872/ psiencia/9.4.21.

9. Montalvo M. Importancia del nivel de motivación laboral y su relación con el perfil profesional de las enfermeras de la micro red - DISA Lima Sur [Tesis para optar el grado académico de maestro de gestión en salud]. Lima: Universidad Privada Norbert Wiener; 2017.
10. Ministerio de Salud. Metodología para el estudio del clima organizacional: Documento técnico. Lima: Ministerio de Salud-Comité Técnico de Clima Organizacional. 2009.

11. Malvarez M, Castrillón M. Panorama de la fuerza de trabajo en enfermería en América Latina. Washington: Unidad de Desarrollo de Recursos Humanos (HR). OPS. 2005.

12. Manosalvas C, Manosalvas L, Nieves J. El clima organizacional y la satisfacción laboral: un análisis cuantitativo riguroso de su relación. AD-minister. 2018; 1(26): 5-15

13. Uceda I. Clima laboral y Satisfacción laboral del usuario interno en el centro de salud Atusparias-Chiclayo, 2017 [Tesis para obtener el grado de maestro en gestión de servicios de salud]. Chiclayo: Escuela de postgrado de la Universidad César Vallejo; 2017.

14. Chiquinta, G. Clima Organizacional y Satisfacción laboral de las enfermeras en el servicio de Emergencia del hospital Regional Lambayeque.Chiclayo-2017 [Tesis para obtener el grado de maestro en gestión de servicios de salud]. Chiclayo: Escuela de posgrado de la Universidad Cesar Vallejo; 2017.

15. Ministerio de Salud. Metodología para el estudio del clima organizacional: Documento técnico. Lima: Ministerio de Salud-Comité Técnico de Clima Organizacional. 2011.

16. Gutiérrez, A. Influencia de los factores motivacionales y de higiene según Herzberg en el clima organizacional del supermercado Metro del distrito de Lambayeque 2015 [Tesis]. Chiclayo: Escuela de administración de Empresas, Universidad Católica Santo Toribio de Mogrovejo; 2016.

17. Montoya Y, Muñoz C. Estudio clima organizacional en la ESE Hospital San Jorge Pereira [Tesis para optar el título de ingeniero industrial]. Pereira: Facultad de Ingeniería industrial, Universidad Tecnológica de Pereira; 2010. 